“ (C) 2013 IEEE. Personal use of this material is permitted. Permission from IEEE must be obtained for all other uses, in any current or future media, including

reprinting/republishing this material for advertising or promotional purposes, creating new collective works, for resale or redistribution to servers or lists, or reuse of any copyrighted component of this work in other works." 


\title{
Observer-based Integral Sliding Mode Control for Sensorless PMSM Drives using FPGA Technology
}

\author{
Nguyen Khanh Quang ${ }^{a}$, Doan Quang Vinh ${ }^{b}$, Nguyen D. That ${ }^{a}$ and Q. P. Ha ${ }^{a}$
}

\begin{abstract}
This paper presents the design and evaluation of an observer-based integral sliding mode controller for sensorless Permanent Magnet Synchronous Motor (PMSM) drive based on the Field Programmable Gate Array (FPGA) technology. For enhancement of robustness, a flux angle estimator mainly constructed by an improved sliding mode observer is utilized to estimate the current and back electromotive force (EMF) as well as to derive the flux angle. These estimated values together with the computed rotor speed of the motor are fed back for the control purpose in both the current loop and the speed loop. To increase the performance of PMSM speed control, an integral sliding mode control (ISMC) is designed with integral operation to improve steady state performance against parameter variations and external disturbances. The developed controller has been implemented in an FPGA-based environment and the very high speed integrated circuit-hardware description language (VHDL) is adopted to show advantages of the proposed control system. By integrating the observer-based and integral sliding mode control techniques into speed control of a PMSM drive, the system performance can be substantially enhanced while improving its cost-effectiveness and reliability. The validity of the proposed approach is verified through simulation results based on the VHDL Modelsim and Simulink co-simulation method.
\end{abstract}

\section{INTRODUCTION}

Due to the advantages of superior power density and high performance motion control with fast speed and enhanced accuracy, the permanent magnet synchronous motors (PMSM) have been increasingly used in robotics, precision machining and many automation processes. As an important application of PMSM, the conventional PMSM control requires precision position data includes rotor position and rotor speed for an efficient field oriented vector control [1-3]. Normally, rotor position can be measured by a physical sensor or by an optical encoder. However, these sensors presents some disadvantages affecting the cost, reliability, noise immunity, and restricted under bad conditions as humidity and corrosion. In recent years, a sensorless control for PMSM drive become an important issue and various sensorless control techniques have been investigated [6-10]. Available sensorless methods are mainly based on the back EMF, sliding mode observer (SMO), Extend Kalmam filter (EKF), the neural network, etc. which require to be able to implement by the fix-pointed processor.

In industrial applications, there are many uncertainties, such as system parameters variation, external load distur-

\footnotetext{
a Faculty of Engineering and Information Technology, University of Technology Sydney, NSW 2007, Australia

${ }^{b}$ Faculty of Electrical Engineering, Danang University of Technology, Danang, Vietnam

Corresponding Email: nkquang@hueic.edu.vn
}

bances, unmodeled uncertainties, etc. which always diminish the performance quality of the pre-design specification in the motor driving system. Although the proportional-integral (PI) controllers have been widely used in in PMSM servo system due to their simple implementation and robustness, it is not easy to obtain a desired performance in the entire operating rage. To this end, intelligent control techniques, such as fuzzy control, neural networks control, sliding mode control, have been developed and applied to the speed control of servo motor drives to achieve high operating performance. The sliding mode control (SMC) is a very useful non linear control method due to its good robustness for parameters variation, external disturbances, fast response as well as simple implementation. To construct a common sliding surface, the sliding mode speed control requires both speed and acceleration signals. However, it is well known that transforming the sensed or the estimated speed into an acceleration signal is very sensitive to noisy effects and uncertainties of parameters. To cope with this problem, an integral sliding mode control (ISMC) with an integral sliding surface is proposed to regulate the PMSM speed [2-5].

With rapid developments of the system-on-chip, field programmable gate array (FPGA) with programmable hardwired feature, fast computation ability, shorter design cycle, embedding processor, low power consumption and higher density becomes an alternative solution. FPGA based controllers have been successfully implemented in many research areas including motion control and PMSM sensorless speed drive. In this paper, we present the design of the proposed controller based on the FPGA technology. To save the resource usage, a sequential finite state machine (FSM) of multipliers, adders, comparators, registers and a look-up table is used [4], [6-8]. Simulation is performed by using the Modelsim/Simulink co-simulation method [4], [7], [10]. The results have verified the effectiveness of the proposed FPGA-based sensorless speed controller for PMSM drives.

\section{SYSTEM DESCRIPTION AND SENSORLESS SPEED CONTROL DESIGN}

The architecture of the proposed speed control system for a sensorless PMSM drive is shown in Fig. 1, where the modelling of PMSM, flux angle and rotor speed estimation, and the speed ISMC are described in this section. 


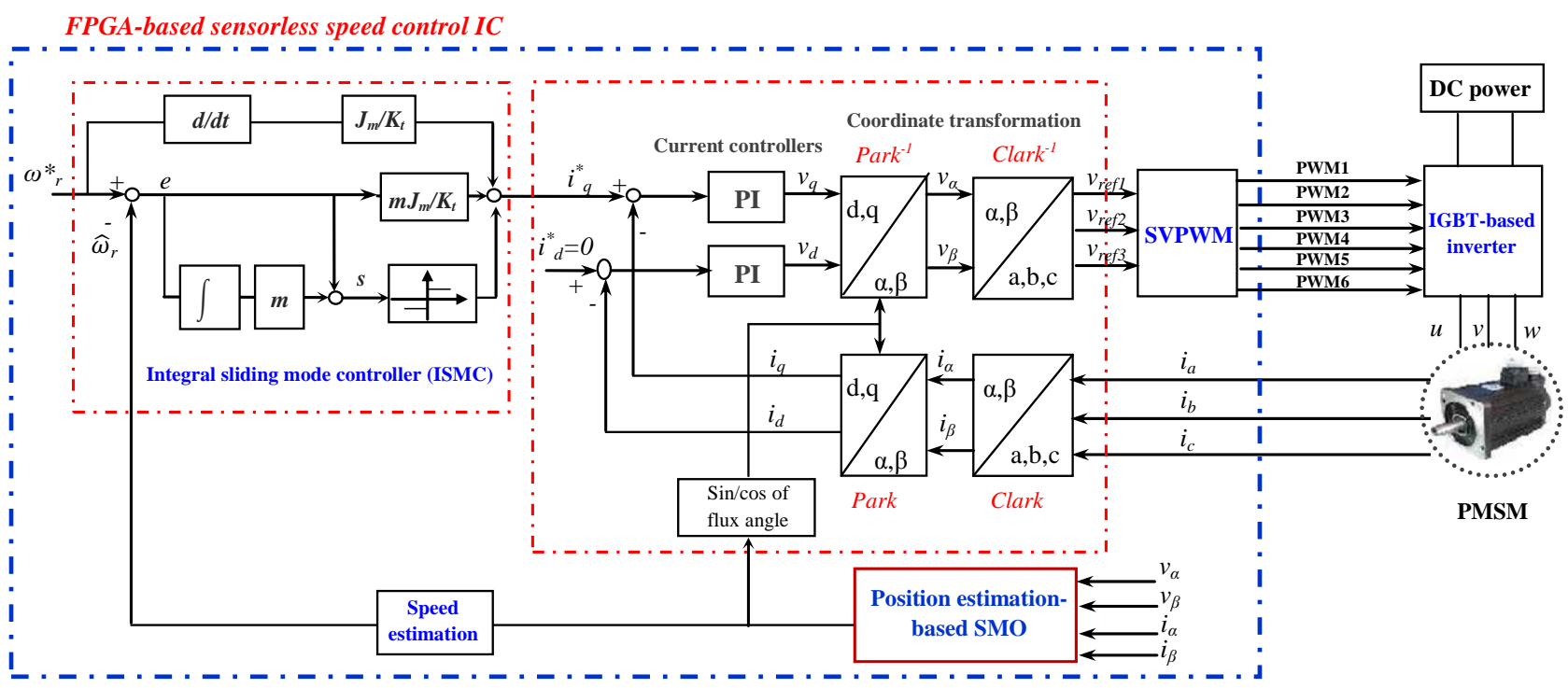

Fig. 1. The proposed speed control system for a sensorless PMSM drive

\section{A. PMSM drive model}

The typical model of PMSM is expressed in $d-q$ synchronous rotating reference frame, as follows

$$
\begin{aligned}
& \frac{d i_{d}}{d t}=-\frac{r_{s}}{L_{s}} i_{d}+\omega_{e} i_{q}+\frac{1}{L_{s}} v_{d} \\
& \frac{d i_{q}}{d t}=-\omega_{e} i_{d}+-\frac{r_{s}}{L_{s}} i_{q}+\frac{1}{L_{s}} v_{q}-\omega_{e} \frac{\lambda_{m}}{L_{s}}
\end{aligned}
$$

where $L_{s} \triangleq L_{d}=L_{q}$ with $L_{d}, L_{q}$ are the $d$ and $q$ axis inductance; $v_{d}, v_{q}$ are the $d$ and $q$ axis voltages; $i_{d}, i_{q}$, are the $d$ and $q$ axis currents, $r_{s}$ is the phase winding resistance; $\omega_{e}$ is the rotating speed of magnet flux; $\lambda_{m}$ is the permanent magnet flux linkage.

The current loop control of PMSM drive in Fig.1 is based on a vector control approach which will control the $i_{d}$ to 0 and decouple the nonlinear model of PMSM to a linear system. Therefore, after decoupling, the torque of PMSM can be written as the following equation,

$$
T_{e}=\frac{3 p \lambda_{m}}{2} i_{q} \triangleq K_{t} i_{q}
$$

Considering the mechanical load, the overall dynamic equation of PMSM drive system is obtained by

$$
T_{e}-T_{L}=J_{m} \frac{d}{d t} \omega_{r}+B_{m} \omega_{r}
$$

where $T_{e}$ is the motor torque, $p$ is pole pairs, $K_{t}$ is torque constant, $J_{m}$ is the inertial value, $B_{m}$ is damping ratio, $T_{L}$ is the external torque, $\omega_{r}$ is rotor speed.

\section{B. Rotor flux position Estimation-based Sliding Mode Ob- server $(S M O)$}

Based on conventional SMO, rotor flux position estimation can be constructed by using a current observer, a onoff controller, a low pass filter and an algorithm position calculation as shown in Fig. 3. The detailed observer design is described as follows.
The circuit equation of PMSM on the $d-q$ rotating coordinate in (1) and (2) is re-formulated as

$$
\left[\begin{array}{l}
v_{d} \\
v_{q}
\end{array}\right]=\left[\begin{array}{cc}
r_{s}+s L_{d} & -\omega_{e} L_{q} \\
\omega_{e} L_{q} & r_{s}+s L_{d}
\end{array}\right]\left[\begin{array}{c}
i_{d} \\
i_{q}
\end{array}\right]+\left[\begin{array}{c}
0 \\
\omega_{e} \lambda_{f}
\end{array}\right],
$$

where $L_{s} \triangleq L_{d}=L_{q}$. Transforming (1) to the one on the $\alpha-$ $\beta$ fixed coordinate, equation (2) can be derived as follows

$$
\left[\begin{array}{l}
v_{\alpha} \\
v_{\beta}
\end{array}\right]=\left[\begin{array}{cc}
r_{s}+s L_{s} & 0 \\
0 & r_{s}+s L_{s}
\end{array}\right]\left[\begin{array}{c}
i_{\alpha} \\
i_{\beta}
\end{array}\right]+\omega_{e} \lambda_{m}\left[\begin{array}{c}
-\sin \theta_{e} \\
\cos \theta_{e}
\end{array}\right],
$$

where $\left[\begin{array}{ll}v_{\alpha} & v_{\beta}\end{array}\right]^{T}$ and $\left[\begin{array}{ll}i_{\alpha} & i_{\beta}\end{array}\right]^{T}$ is the voltage and current in fixed coordinates, $\theta_{e}$ is the angular position at the magnet flux and $s$ is differential operator. In addition, let us define the EMF as

$$
e=\left[\begin{array}{c}
e_{\alpha} \\
e_{\beta}
\end{array}\right] \triangleq \omega_{e} \lambda_{m}\left[\begin{array}{c}
-\sin \theta_{e} \\
\cos \theta_{e}
\end{array}\right]
$$

The EMF includes the position information from the flux. By estimating the EMF, it is possible to get $\theta_{e}$ from its phase.

To easily observe the EMF, (2) is rewritten as the state space form by current variable,

$$
\frac{d}{d t}\left[\begin{array}{l}
i_{\alpha} \\
i_{\beta}
\end{array}\right]=-\frac{r_{s}}{L_{s}} \cdot\left[\begin{array}{l}
i_{\alpha} \\
i_{\beta}
\end{array}\right]+\frac{1}{L_{s}}\left[\begin{array}{l}
v_{\alpha} \\
v_{\beta}
\end{array}\right]-\frac{1}{L_{s}}\left[\begin{array}{l}
e_{\alpha} \\
e_{\beta}
\end{array}\right] .
$$

A sliding mode observer is designed by

$$
\frac{d}{d t}\left[\begin{array}{l}
\hat{i}_{\alpha} \\
\hat{i}_{\beta}
\end{array}\right]=-\frac{r_{s}}{L_{s}} \cdot\left[\begin{array}{l}
\hat{i}_{\alpha} \\
\hat{i}_{\beta}
\end{array}\right]+\frac{1}{L_{s}}\left[\begin{array}{c}
v_{\alpha} \\
v_{\beta}
\end{array}\right]-\frac{1}{L_{s}}\left[\begin{array}{c}
z_{\alpha} \\
z_{\beta}
\end{array}\right]
$$

where the $\left[\begin{array}{ll}\hat{i}_{\alpha} & \hat{i}_{\beta}\end{array}\right]^{T}$ is the estimated current on fixed coordinate and the $\mathrm{Z}$ is the output gain of the switching controller. In conventional $\mathrm{SMO}$, the $\mathrm{Z}$ is defined as

$$
Z=\left[\begin{array}{c}
z_{\alpha} \\
z_{\beta}
\end{array}\right] \triangleq k \cdot \operatorname{sgn}\left(\left[\begin{array}{c}
\hat{i}_{\alpha}-i_{\alpha} \\
\hat{i}_{\beta}-i_{\beta}
\end{array}\right]\right),
$$


To solve the chattering problem, the sign function is replaced by a saturation function shown in Fig. 2 and defined as:

$$
\operatorname{sat}\left(\frac{s}{\xi}\right)= \begin{cases}\frac{s}{\xi} & \left|\frac{s}{\xi}\right| \leq 1 \\ \operatorname{sgn}\left(\frac{s}{\xi}\right) & \left|\frac{s}{\xi}\right|>1\end{cases}
$$

and the constant factor $\xi$ defines the thickness of the boundary layer.

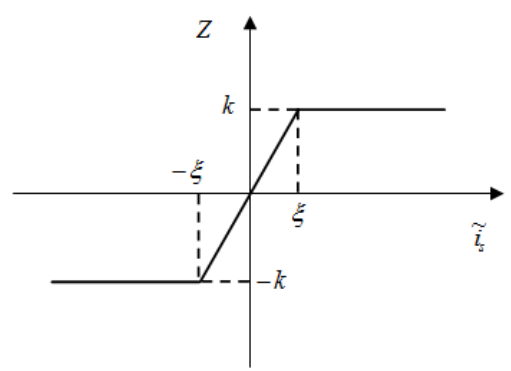

Fig. 2. Diagram of the saturation function

The dynamic estimation error is obtained by subtracting (5) from (4) as

$$
\frac{d}{d t}\left[\begin{array}{c}
\tilde{i}_{\alpha} \\
\tilde{i}_{\beta}
\end{array}\right]=-\frac{r_{s}}{L_{s}} \cdot\left[\begin{array}{c}
\tilde{i}_{\alpha} \\
\tilde{i}_{\beta}
\end{array}\right]+\frac{1}{L_{s}}\left[\begin{array}{c}
e_{\alpha} \\
e_{\beta}
\end{array}\right]-\frac{1}{L_{s}}\left[\begin{array}{c}
z_{\alpha} \\
z_{\beta}
\end{array}\right]
$$

where the sliding surface is defined by $e_{i} \triangleq\left[\begin{array}{cc}\tilde{i}_{\alpha} & \tilde{i}_{\beta}\end{array}\right]^{T}=$ $\left[\begin{array}{ll}\hat{i}_{\alpha}-i_{\alpha} & \hat{i}_{\beta}-i_{\beta}\end{array}\right]^{T}$. Further, if we choose the $\mathrm{k}$ be large enough, i.e, $k \geq \max \left(\left|e_{\alpha}\right|,\left|e_{\beta}\right|\right)$, the inequality $e_{i}^{T} \cdot \dot{e}_{i}<0$ can be reached and the SMO can enter into sliding mode condition, i.e, $e_{i}=\dot{e}_{i}=0$. The $\mathrm{Z}$ in (8) will approach to EMF

$$
\left[\begin{array}{c}
z_{\alpha} \\
z_{\beta}
\end{array}\right]=\left[\begin{array}{c}
e_{\alpha} \\
e_{\beta}
\end{array}\right]=\omega_{e} \lambda_{f}\left[\begin{array}{c}
-\sin \theta_{e} \\
\cos \theta_{e}
\end{array}\right] .
$$

To alleviate the high frequency in switching control, a lowpass filter is applied.

$$
\frac{d}{d t}\left[\begin{array}{l}
\hat{e}_{\alpha} \\
\hat{e}_{\beta}
\end{array}\right]=-\omega_{o} \cdot\left[\begin{array}{l}
\hat{e}_{\alpha} \\
\hat{e}_{\beta}
\end{array}\right]+\omega_{o}\left[\begin{array}{c}
z_{\alpha} \\
z_{\beta}
\end{array}\right]
$$

where $\omega_{o}=2 \pi f_{o}$. Finally, the rotor position $\hat{\theta}_{e}$ can be computed by

$$
\hat{\theta}_{e}=\tan ^{-1}\left(-\frac{\hat{e}_{\alpha}}{\hat{e}_{\beta}}\right)
$$

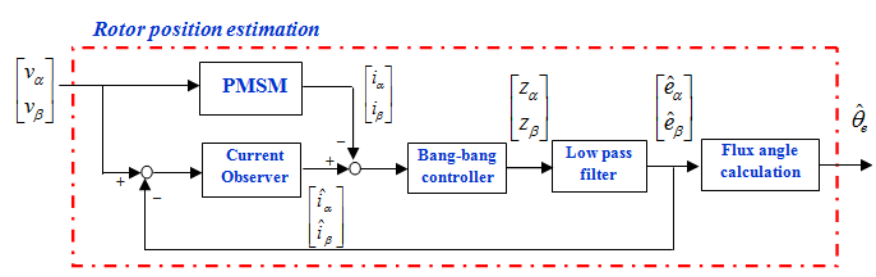

Fig. 3. Rotor flux angle estimation based on the conventional SMO

\section{Integral Sliding Mode Control Design in Speed Loop}

From (4), the torque equation of PMSM system can be derived as

$$
\frac{d}{d t} \omega_{r}=\frac{3 p \lambda_{m}}{2 J_{m}} i_{q}-\frac{B_{m}}{J_{m}} \omega_{r}-\frac{T_{L}}{J_{m}}=\frac{3 p \lambda_{m}}{2 J_{m}} i_{q}^{*}+\mu(t)
$$

where $\mu(t)=\frac{3 p \lambda_{m}}{2 J_{m}}\left(i_{q}-i_{q}^{*}\right)-\frac{B_{m}}{J_{m}} \omega_{r}-\frac{T_{L}}{J_{m}}$ is the lumped disturbances of system. Define the speed error $e=\omega_{r}^{*}-\omega_{r}$ where $\omega_{r}^{*}$ is reference speed. Taking the derivative of $e$ and substituting (16) into it, yields:

$$
\dot{e}=\dot{\omega}_{r}^{*}-\frac{3 p \lambda_{m}}{2 J_{m}} i_{q}^{*}-\mu(t)
$$

The sliding surface is defined with integral component as

$$
s=e+m \int_{0}^{t} e d \tau
$$

and the variable structure speed controller is designed as follows

$$
i_{q}^{*}=\frac{2 J_{m}}{3 p \lambda_{m}} m \cdot e+\frac{2 J_{m}}{3 p \lambda_{m}} \cdot \dot{\omega}_{r}^{*}+k_{s} \cdot \operatorname{sign}(s),
$$

where $m, k_{s}>0$, and $\operatorname{sign}($.$) is the signum function.$

Assume that the lumped disturbances of the system $\mu(t)$ satisfies $0 \leq|\mu(t)|<d$. Choosing Lyapunov function $V=$ $\frac{1}{2} s^{2}$, and taking the derivative of it along system (18), yields:

$$
\dot{V}=s . \dot{s}=s[\dot{e}+m e]<0
$$

Substituting (17) into (20), the stability condition can be represented with

$$
\begin{aligned}
\dot{V} & =s\left[\dot{\omega}_{r}^{*}-\frac{3 p \lambda_{m}}{2 J_{m}} i_{q}^{*}-\mu(t)+m e\right]<0 \\
& =-\frac{3 p \lambda_{m}}{2 J_{m}} s\left[i_{q}^{*}-\frac{2 J_{m}}{3 p \lambda_{m}} m e-\frac{2 J_{m}}{3 p \lambda_{m}} \dot{\omega}_{r}^{*}+\frac{2 J_{m}}{3 p \lambda_{m}} \mu(t)\right]<0
\end{aligned}
$$

Substituting (19) into (21),

$$
\begin{aligned}
\dot{V} & =-\frac{3 p \lambda_{m}}{2 J_{m}} s\left[k_{s} \cdot \operatorname{sign}(s)+\frac{2 J_{m}}{3 p \lambda_{m}} \mu(t)\right]<0 \\
& =-\frac{3 p \lambda_{m}}{2 J_{m}}\left[k_{s} \cdot \operatorname{sign}(s) \cdot s+\frac{2 J_{m}}{3 p \lambda_{m}} \mu(t) \cdot s\right]<0 \\
& =-\frac{3 p \lambda_{m}}{2 J_{m}}\left[k_{s}|s|+\frac{2 J_{m}}{3 p \lambda_{m}} d \cdot s\right]<0
\end{aligned}
$$

where the switching control gain can be derived to satisfy the inequality condition as follows

$$
k_{s}>\frac{2 J_{m}}{3 p \lambda_{m}} d
$$




\section{FPGA-BASED SENSORLESS SPEED CONTROL IMPLEMENTATION}

\section{A. Controller architecture}

Fig.4 shows the FPGA-based architecture of the proposed sensorless control system for the PMSM drive. The controller includes a ISMC speed controller, a PI current controller and coordinate transformation (CCCT), a SVPWM generation and SMO-based rotor position estimation. All modules presented in Fig.4 are described by Verilog HDL and simulated in Modelsim as well as tested in Altera Cyclone II EP2C70 board. The frequency divider generates $50 \mathrm{MHz}(\mathrm{Clk}), 12.5$ $\mathrm{MHz}$ (Clk-sp) and $16 \mathrm{KHz}$ (Clk-ctr) clock to supply all circuits.

\section{B. Algorithm implementation}

A finite state machine (FSM) is employed to describe the control algorithm of the ISMC and SMO-based rotor position estimation, as shown in Figs. 5 and 6, respectively. In Fig. 5 , the data type adopts a 16-bit length with Q15 format and 2 's complement operations. Although the algorithm of ISMC described is complex, the FSM adequately incorporates the control structure and can be easily described by VHDL. The multiplier and adder apply the Altera LPM (Library Parameterized Modules) standard. It manipulates 13 steps machine to carry out the overall computations. The steps $S_{0}-S_{6}$ are for the computation of speed error and sliding function; step $S_{7}$ executes the signum function for switching control; and finally the sliding mode control is realized in steps $S_{8}-S_{13}$. The operation of each step can be completed within 80 ns $(12.5 \mathrm{MHz})$; therefore total 13 steps need 1.04 $\mu s$ for the ISMC operation. The SMO-based rotor position estimation algorithm is shown in Fig. 6. The data type adopts a 12-bit length with Q11 format. The steps $S_{0}-S_{8}$ execute the estimation of currents; steps $S_{9}-S_{10}$ compute the current errors; $S_{11}$ calculate the saturation function for the switching control; steps $S_{12}-S_{16}$ describe the computation of EMF and $S_{17}-S_{36}$ perform the computation of the rotor position. The implementation of SMO needs $2.88 \mu \mathrm{s}$ for 36 steps. Other circuit designs such as CCCT and SVPWM, shown in Fig. 4, can be referred to [4]. Finally, the resource usage of ISMC, CCCT and SMO are 8,601 ALUTs and 92,747 RAM bits resource for the Cyclone II EP2C70.

\section{SIMULATION AND RESULTS}

Simulation is performed by using Electronic Design Automation (EDA) Simulator Link, which provides a verification interface between MATLAB or Simulink and the HDL simulator or FPGA board. The ModelSim performs the function of adaptive PI controller, SMO and current vector controller which is described using VHDL code. In the Simulink, the SimPowerSystem blockset can provide the components of PMSM and the inverter and it also can generate stimuli to ModelSim and analyze the simulations responses. The designed PMSM parameters applied in simulation of Fig.4 are that pole pairs is 4 , stator phase resistance is 1.3 , stator inductance is $6.3 \mathrm{mH}$, inertia is $\mathrm{J}=0.00011 \mathrm{~kg} . \mathrm{m}^{2}$ and friction factor is $\mathrm{F}=0.0014$ N.m.s. In the simulation of sensorless

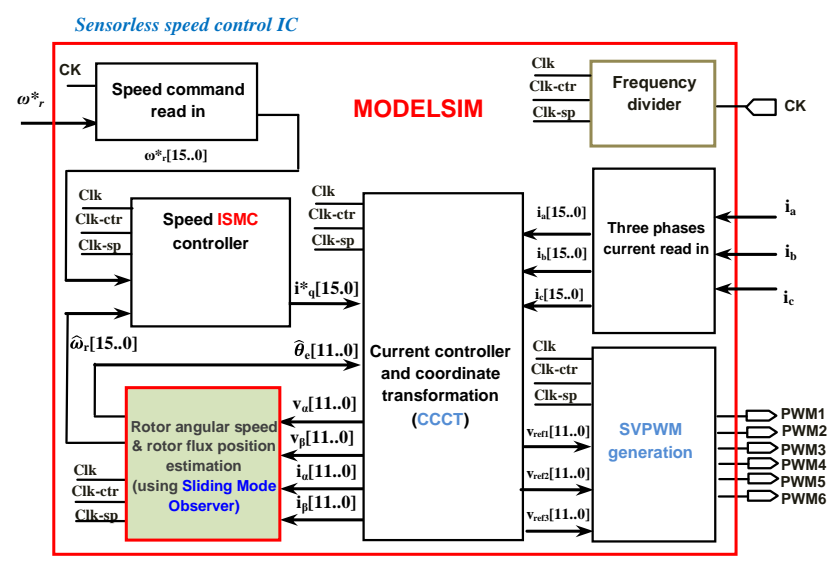

Fig. 4. The proposed speed control system for a sensorless PMSM drive

PMSM drive, rotor position estimation based on SMO is firstly evaluated. The conventional SMO method with signum function and the improved one with saturation function are tested with PMSM running speed at $500 \mathrm{rpm}$ and these simulation results presented in Figs. 7, 8. The results show that the response of the estimated rotor flux position can follow with the actual rotor flux position in both methods. On comparing the results of two methods, it is clearly observed that the chattering of rotor position estimation is reduced and its accuracy is improved significantly in SMO where saturation function is used. Secondly, the performance of sliding mode control using integral operation is verified. Three tested cases are evaluated with parameter variations: Case 1: (Normal-load condition)

$$
J=0.00011, \quad F=0.0014 ;
$$

case 2: (Light-load condition)

$$
J=0.00011 / 3, \quad F=0.0014 / 3
$$

and case 3: (Heavy-load condition)

$$
J=0.00011 * 3, \quad F=0.0014 * 3
$$

The $20 \mathrm{~Hz}$ square wave with magnitude of $500 \mathrm{rpm}$ is used as tested command. When the speed controller is adopted by PI controller only $\left(K_{p}=0.36, K_{i}=0.005\right)$ and the PMSM parameters are initially designed at the normal load condition (case 1), the simulation result is shown in Fig. 9 which present a good following response. However, when the running condition is changed to the light-load condition (case 2) and heavy-load condition (case 3), the results in Figs. 10, 11 show that the step speed response become worse with overshoot in the light-load condition and slow response occurred in heavyload condition. It demonstrates that although the sensorless control based on SMO in PMSM drive can give a good speed tracking, it is still easily affected by external load variation. To cope with this problem, an integral SMC is adopted in Fig. 1. The ISMC has an integral sliding surface to reject the requirement of the acceleration signal, which is usual in traditional sliding-mode speed control techniques. Due to the nature of the sliding control, this control scheme is 


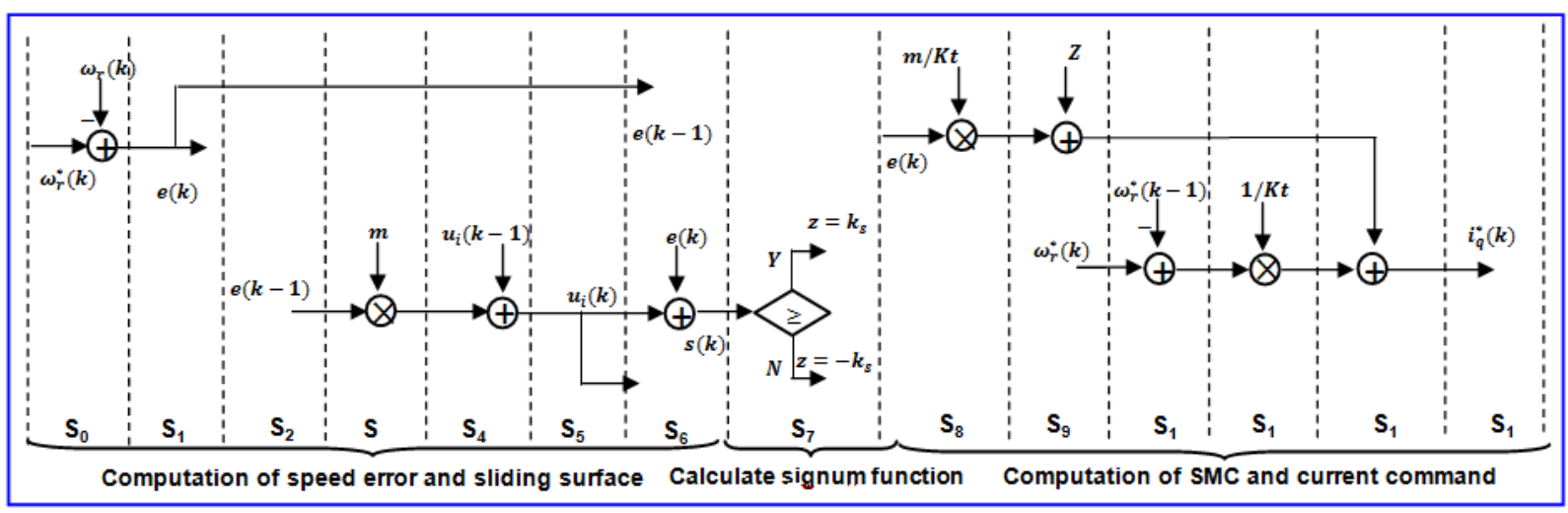

Fig. 5. State diagram of an FSM for the speed controller using ISMC

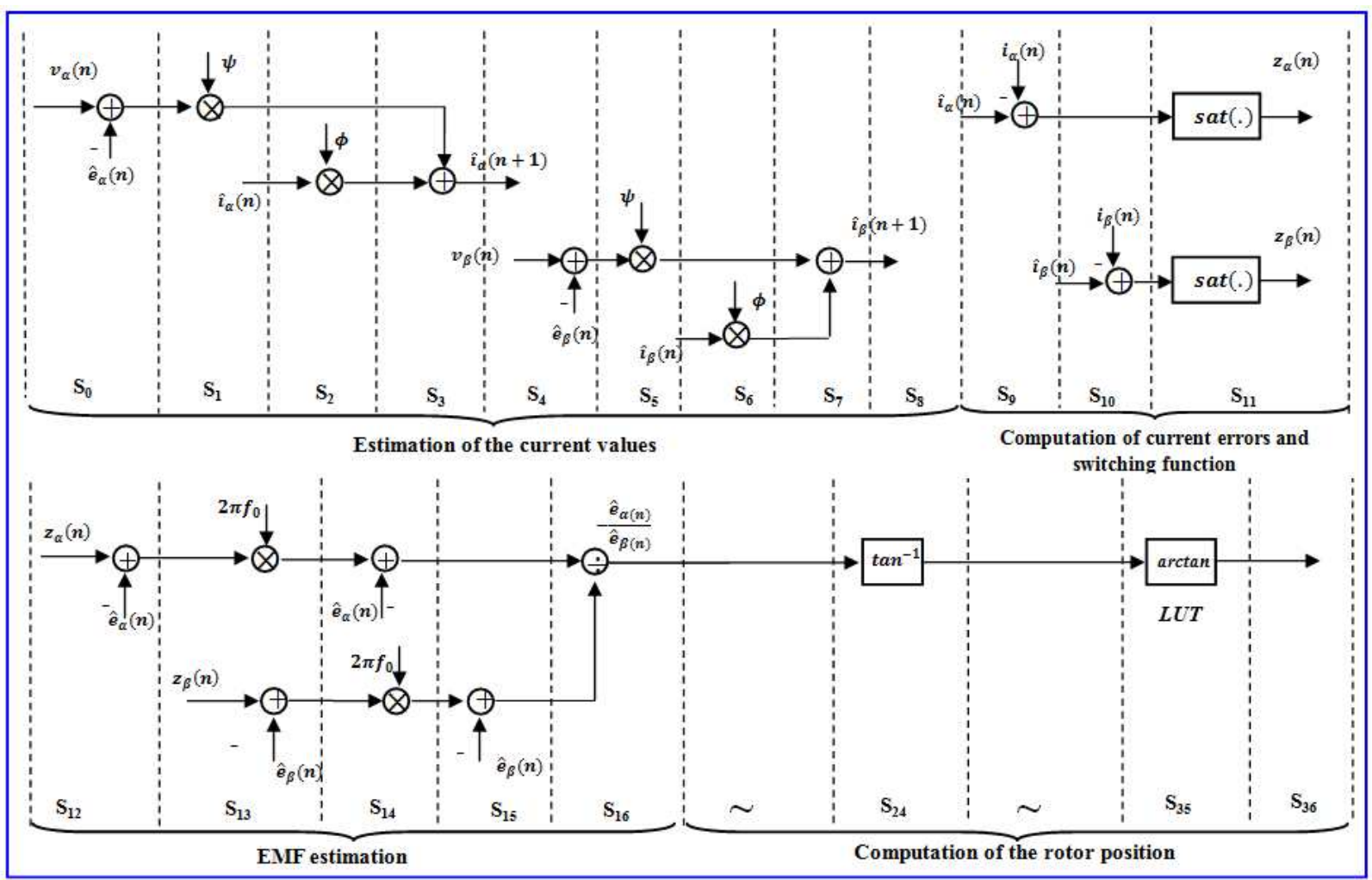

Fig. 6. State diagram of an FSM for an improved SMO-based rotor position estimation algorithm

robust under uncertainties caused by parameter variations or by changes in the external load. Figs. 12 and 13 show the simulation results while it uses the proposed ISMC control in sensorless PMSM drive. The results show an improvement which rotor speed response no overshoot and less rising time in both case 1 and 2 . These also present that the proposed ISMC can enhance robustness in sensorless PMSM drive.

\section{CONCLUSIONS}

In this paper, a FPGA-based sensorless sliding-mode vector control has been presented. The proposed system comprises a sliding mode observer, a field-oriented PI current controller, and an ISMC for the speed control loop. All the system architecture can be integrated and realized in one
FPGA chip. The SMO is used to estimate the rotor position and speed of the PMSM due to its strong robustness, the sign function is replaced by the saturation function to reduce system chattering, and the traditional SMO is improved. In addition, a sliding-mode controller with an integral switching surface is proposed. This control scheme is robust under uncertainties. Simulation results validate the feasibility and effectiveness of the proposed control system.

\section{REFERENCES}

[1] V.D. Colli and R.D. Stefano and F. Marignetti, "A system-on-chip sensorless control for a permanent magnet synchronous motor," IEEE Transactions on Industrial Electronics, vol. 57, no. 11, pp. 3822-3829, Nov. 2010. 
[2] W. Xiang and F. Chen, "Sliding mode control strategies for the hyperchaotic MCK system," Express Letters, vol. 3, no. 3, pp. 283288, 2009.

[3] C.Y. Chen, "Sliding mode controller design of induction motor based on space-vector pulsewidth modulation method," International Journal of Innovative Computing, Information and Control, vol. 5, no. 10, pp. 3603-3614, 2009.

[4] N. K. Quang, , N. D. That; N. H. Quang, Q. P. Ha, "FPGA-based fuzzy sliding mode control for sensorless PMSM drive," 2012 IEEE International Conference on Automation Science and Engineering, (CASE), pp.172-177, Aug. 2012

[5] O. Barambones, A.J. Garrido, F.J. Maseda, "Integral sliding-mode controller for induction motor based on field-oriented control theory," Control Theory and Applications IET, vol. 1, no. 3, pp. 786-794, May 2007.

[6] K. Hongryel, S. Jubum, and L. Jangmyung, "A High-Speed SlidingMode Observer for the Sensorless Speed Control of a PMSM," IEEE Transactions on Industrial Electronics, vol. 58, pp. 4069-4077, 2011.

[7] N. K. Quang, N. T. Hieu, G. P. Hunter, and Q. P. Ha, "FPGA-based Sensorless PMSM Drive Using Parallel Reduced-Order Extended Kalman Filter,' Proc. 2012 IEEE International Conference on Control, Automation and Information Sciences, (ICCAIS), pp. 164-169, Nov. 2012.

[8] L. Idkhajine, E. Monmasson, and A. Maalouf, "Fully FPGA-Based Sensorless Control for Synchronous AC Drive Using an Extended Kalman Filter," Industrial Electronics, IEEE Transactions on, vol. 59, pp. 3908-3918, Oct. 2012.

[9] E. Monmasson, L. Idkhajine, M. N. Cirstea, I. Bahri, A. Tisan, and M. W. Naouar, "FPGAs in Industrial Control Applications," Industrial Informatics, IEEE Transactions on, vol. 7, no. 2, pp. 224-243, May 2011.

[10] Y. Li , J. Huo, X. Li, J. Wen, Y. Wang and B. Shan, "An openloop sin microstepping driver based on FPGA and the Co-simulation of Modelsim and Simulink", Proc. International Conference on Computer, Mechatronics, Control and Electronic Engineering, (CMCE), pp. 223-227, 2010.

[11] Matlab/Simulink Users Guide, Application Program Interface Guide, The Mathworks, 2004.

[12] D.-H. Yim, B.-G. Park and D.-S. Hyun, "Sensorless control strategy of IPMSM based on a parallel reduced-order EKF," Proc. IECON 2010 36th Annual Conference on IEEE Industrial Electronics Society, pp. 2258-2263, 2010.

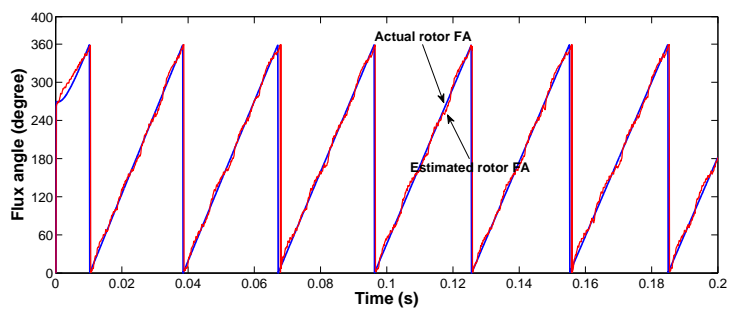

Fig. 7. Flux angle (FA) waveforms obtained by the conventional SMO method using the signum function

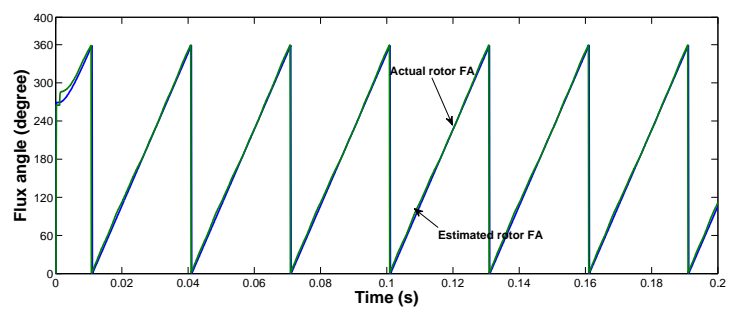

Fig. 8. Flux angle (FA) waveforms obtained by the improved SMO method using the saturation function
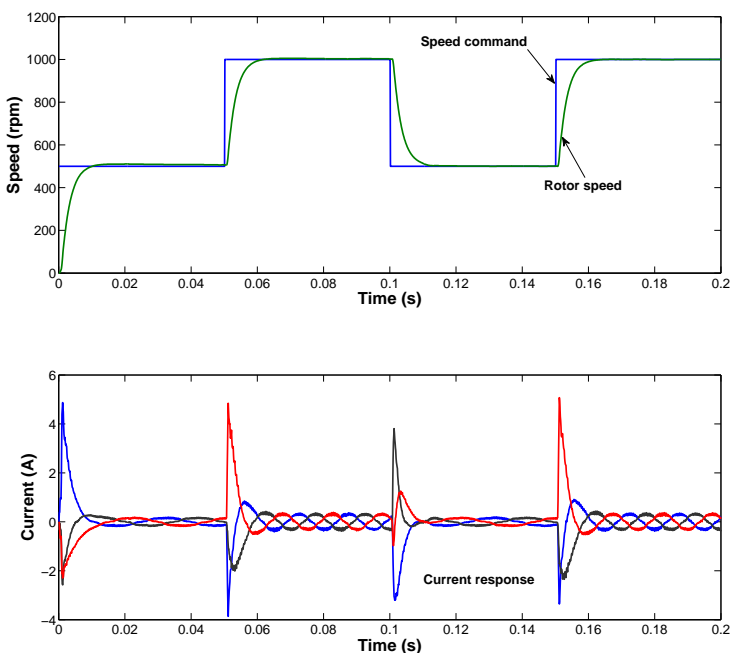

Fig. 9. Simulation result when PI controller is used while sensorless PMSM operated at normal load condition
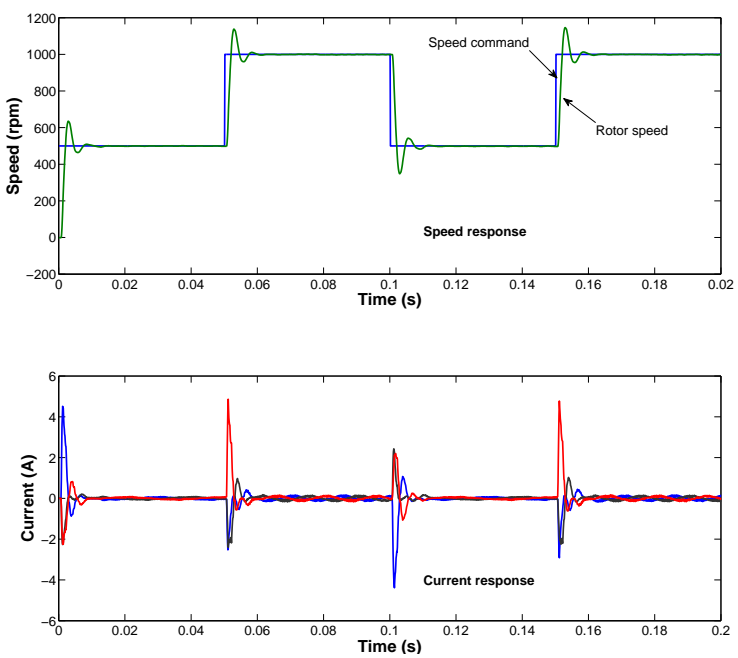

Fig. 10. Simulation result when PI controller is used while sensorless PMSM operated at light load condition
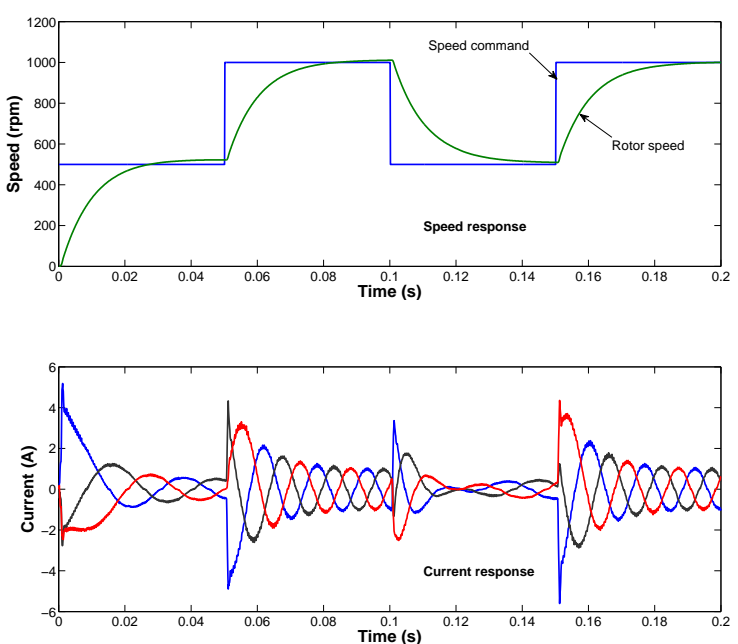

Fig. 11. Simulation result when PI controller is used while sensorless PMSM operated at heavy load condition 

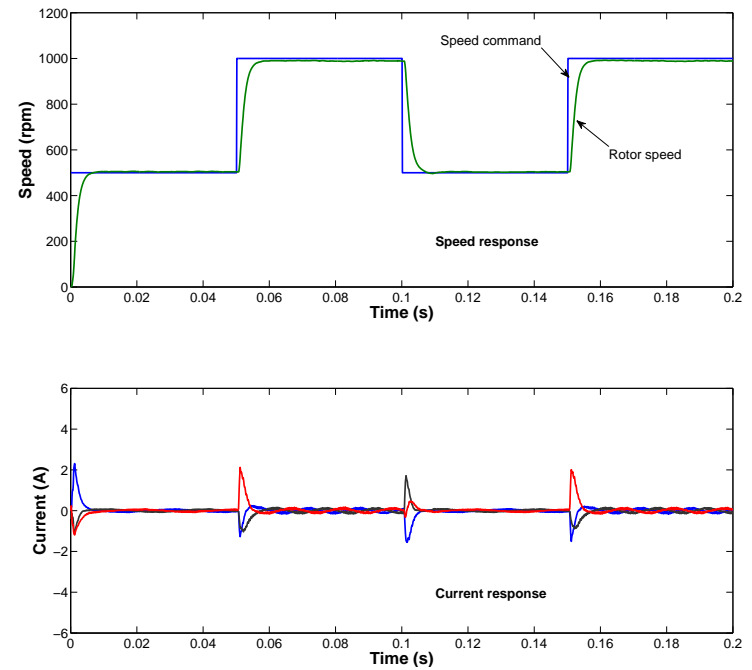

Fig. 12. Simulation result when ISMC controller is used while sensorless PMSM operated at light load condition
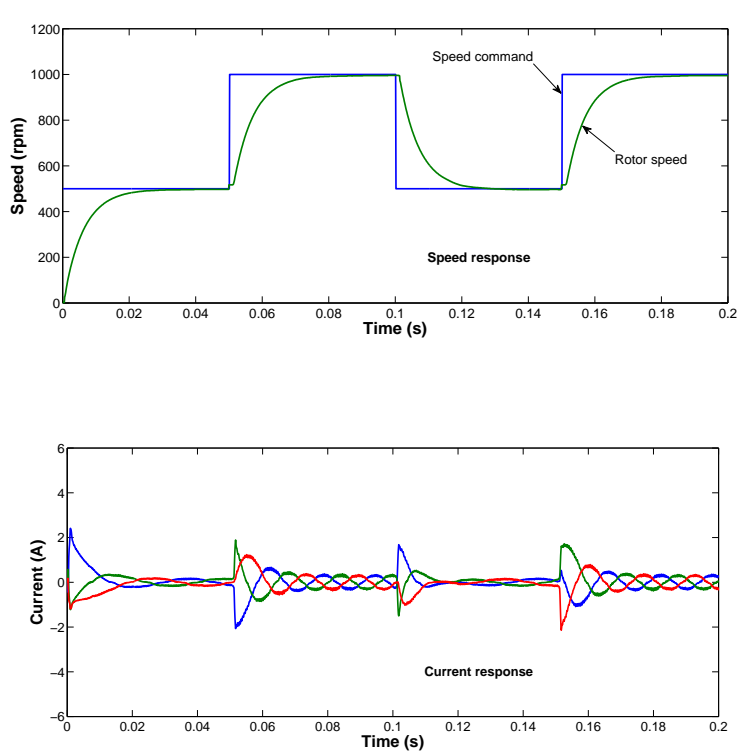

Fig. 13. Simulation result when ISMC controller is used while sensorless PMSM operated at heavy load condition 